\title{
Validation of the mutant selection window hypothesis with fosfomycin against Escherichia coli and Pseudomonas aeruginosa: an in vitro and in vivo comparative study
}

\begin{abstract}
Ai-jun Pan ${ }^{1,2}$, Qing $\mathrm{Mei}^{2}$, Ying Ye ${ }^{1,3,4}$, Hong-ru $\mathrm{Li}^{5}$, Bao $\mathrm{Liu}^{2}$ and Jia-bin $\mathrm{Li}^{1,3,4,6}$
The purpose of this study was to validate the mutant selection window (MSW) hypothesis in vitro and in vivo with Escherichia coli and Pseudomonas aeruginosa exposed to fosfomycin. Two standard strains of Gram-negative bacteria, those are E. coli ATCC 25922 and $P$. aeruginosa ATCC 27853, were exposed to fosfomycin at concentrations below MIC, between the MIC and the mutant prevention concentration (MPC), and above the MPC in Luria-Bertani broth and in a tissue-cage infection model, respectively. With the in vitro time-kill studies, there were bacterial re-growth and emergence of resistance thereafter for both strains at antibiotic concentrations of $\times 4, \times 8$ and $\times 16$ MIC. In our animal model, the loss in susceptibility of $P$. aeruginosa at fosfomycin concentrations fluctuated between the lower and upper boundaries of the MSW. In contrast, the emergence of resistant mutants of $E$. coli was not observed in vivo, regardless of fosfomycin dosage. Interestingly, the in vitro-isolated resistant mutants of $E$. coli showed a decreased growth rate compared with the susceptible parental strains, whereas no fitness cost in $P$. aeruginosa was observed. The emergence of antibiotic resistance is shaped by several factors. MSW theory may not apply to all antimicrobial-pathogen combinations. Before it can be used as a framework for the design of antimicrobial therapy, the existence of the window must be demonstrated not only in vitro but also in vivo.
\end{abstract}

The Journal of Antibiotics (2017) 70, 166-173; doi:10.1038/ja.2016.124; published online 19 October 2016

\section{INTRODUCTION}

Fosfomycin is a natural antibacterial agent that has been known for $>40$ years. Oral fosfomycin has been approved by the Food and Drug Administration for the treatment of uncomplicated urinary tract infections (UTIs) in adult women that is caused by Escherichia coli. ${ }^{1,2}$ The rapid emergence and spread of multidrug-resistant pathogens including Pseudomonas aeruginosa has resulted in revived interest in the parenteral use of fosfomycin to treat infections other than UTIs. ${ }^{3-6}$ It is generally well tolerated at therapeutic doses, with a low incidence of adverse events. However, clinicians use the medication mainly as a combination therapy, because fosfomycin resistance occurs rapidly with monotherapy. ${ }^{6}$

Resistant subpopulations are proposed to be selectively enriched in a specific range called the mutant selection window (MSW), the concentration range between MIC and mutant prevention concentration (MPC). ${ }^{7}$ MPC is defined as the lowest antibiotic concentration that prevents the growth of resistant mutants with a density of inoculum $\geqslant 10^{9} \mathrm{CFU} \mathrm{ml}^{-1} .8 \mathrm{MSW}$ hypothesis has been validated with different antimicrobial-pathogen combinations in vitro and in vivo. ${ }^{9-12}$ It is generally believed that this hypothesis may have broad applications for fighting the growing problem of antimicrobial resistance, and then the clinical applications of MSW were investigated quite intensively in recent years. ${ }^{13-16}$ However, the applicability of the MSW to most of these antimicrobial-pathogen combinations was investigated only with in vitro model. In previous studies, this concept has also been proven to be relevant for vancomycin, but our in silico simulation results truly questioned the anti-mutant pharmacokinetics/pharmacodynamics (PK/PD) index derived from in vitro experiments. ${ }^{12,17}$ Thus, we believe that results obtained from in vitro experiments cannot usually be reliably extrapolated to in vivo behavior.

In the present study, we tested the MSW hypothesis with fosfomycin, along with an attempt to describe the emergence of fosfomycin resistance in vitro and in vivo. Two standard strains of Gram-negative bacteria, those are E. coli ATCC 25922 and P. aeruginosa ATCC 27853, were exposed to fosfomycin at concentrations below the MIC, between the MIC and MPC and above the MPC in Luria-Bertani broth and in a tissue-cage infection

${ }^{1}$ Department of Infectious Diseases, First Affiliated Hospital of Anhui Medical University, Hefei, Anhui, China; ${ }^{2}$ Department of Critical Care Medicine, Affiliated Provincial Hospital of Anhui Medical University, Hefei, Anhui, China; ${ }^{3}$ Anhui Center for Surveillance of Bacterial Resistance, Hefei, Anhui, China; ${ }^{4}$ Institute of Bacterium Resistance, Anhui Medical University, Hefei, Anhui, China; ${ }^{5}$ Class 2012, The Eight-year Program on Clinical Medicine, Xiangya School of Medicine, Central South University, Changsha, Hunan, China and ${ }^{6}$ Department of Infectious Diseases, Chaohu Hospital affiliated to Anhui Medical University, Chaohu, Anhui, China

Correspondence: Dr J-b Li, Department of Infectious Diseases, The First Affiliated Hospital of Anhui Medical University, Hefei, Anhui 230022, China.

E-mail: lijiabin948@vip.sohu.com

Received 5 June 2016; revised 28 August 2016; accepted 12 September 2016; published online 19 October 2016 
model, respectively. In addition, the emergence of antibiotic resistance in a bacterial population is affected by several factors, of which the biological fitness cost of the mutants has been considered critical. ${ }^{18}$

\section{RESULTS}

MIC, $\mathrm{MIC}_{99}$ and MPC of fosfomycin

Under aerobic conditions, the $\mathrm{MIC}$ and $\mathrm{MIC}_{99}$ of fosfomycin for E. coli ATCC 25922 were estimated at 2 and $1.2 \mu \mathrm{g} \mathrm{ml}^{-1}$, and those for $P$. aeruginosa ATCC 27853 were 4 and $3.6 \mu \mathrm{g} \mathrm{m}^{-1}$, respectively. Under anaerobic conditions, the MIC and $\mathrm{MIC}_{99}$ of fosfomycin for E. coli ATCC 25922 were estimated at 1 and $0.8 \mu \mathrm{g} \mathrm{ml}^{-1}$, and those for $P$. aeruginosa ATCC 27853 were 2 and $1.8 \mu \mathrm{g} \mathrm{ml}^{-1}$, respectively. The exact MPC of fosfomycin $E$. coli and $P$. aeruginosa were estimated at 57.6 and $102.4 \mu \mathrm{g} \mathrm{ml}^{-1}$ under both aerobic and anaerobic conditions, respectively.

\section{Mutation frequency to resistance in vitro}

Table 1 shows the frequencies of spontaneous single-step mutations leading to fosfomycin resistance. Both of these two strains had a high mutation frequency to fosfomycin resistance in vitro and exhibited lower frequency of mutants to fosfomycin under anaerobic compared with aerobic conditions. E. coli ATCC 25922 compared with $P$. aeruginosa ATCC 27853 exhibited lower frequencies of mutants to fosfomycin under both aerobic and anaerobic conditions as summarized in Table 1. MIC values of all spontaneous mutants increased 8- to 32-fold for fosfomycin.

\section{Bactericidal activities and emergence of resistance in vitro}

Figure 1 shows the representative survival curves against E. coli ATCC 25922 and P. aeruginosa ATCC 27853 under aerobic and anaerobic conditions. Overall, killing was more rapid under anaerobic conditions. For both strains, bacterial counts increased to ca. $10^{9}$ $\mathrm{CFU} \mathrm{ml} \mathrm{m}^{-1}$ after $24 \mathrm{~h}$ without antimicrobials and remained unchanged at $\times 0.5$ aerobic MIC under both conditions. At antibiotic concentrations of $\times 4, \times 8$ and $\times 16$ aerobic MIC, fosfomycin did not exhibit rapid bactericidal activity against high-inoculum planktonic bacteria under either aerobic or anaerobic conditions; there was a temporary inhibitory effect until 12 or $24 \mathrm{~h}$ and then both the strains quickly re-grew; moreover, the mutants of E. coli and $P$. aeruginosa with increased MIC were readily selected at the end point of the aerobic and anaerobic time-kill studies. The MICs for all mutants increased to $64-128 \mu \mathrm{g} \mathrm{ml}^{-1}$ and the observed elevations in the MIC were stable after ten passages on antibiotic-free agar plates.

Table 1 Frequency of spontaneous Escherichia coli ATCC 25922 and Pseudomonas aeruginosa ATCC 27853 mutants with increased fosfomycin MIC under aerobic and anaerobic conditions at $4 \times, 8 \times$ and $16 \times \mathrm{MIC}$

\begin{tabular}{lccc}
\hline & \multicolumn{3}{c}{ Fold MIC } \\
\cline { 2 - 4 } & $\times 4$ & $\times 8$ & $\times 16$ \\
Strains and condition & & & \\
\hline $\begin{array}{l}\text { E. coli ATCC 25922 } \\
\quad \text { Aerobic }\end{array}$ & $8 \times 10^{-6}$ & $8 \times 10^{-6}$ & $6 \times 10^{-7}$ \\
$\quad$ Anaerobic & $2 \times 10^{-6}$ & $2 \times 10^{-6}$ & $1 \times 10^{-7}$ \\
P. aeruginosa ATCC 27853 & & & \\
$\quad$ Aerobic & $6 \times 10^{-6}$ & $4 \times 10^{-6}$ & $2 \times 10^{-6}$ \\
$\quad$ Anaerobic & $1 \times 10^{-6}$ & $1 \times 10^{-6}$ & $8 \times 10^{-7}$ \\
\hline
\end{tabular}

aFinal concentration used for calculation of mutant frequency.
At both the lowest $(\times 0.5$ aerobic MIC) and the highest $(\times 32$ aerobic MIC) concentrations, no development of resistance to fosfomycin was observed under either aerobic or anaerobic conditions.

\section{Effect of fosfomycin dose on bacterial survival in the tissue-cage model}

When ca. $3.0 \times 10^{10}$ bacteria were injected into an implanted ball, 6 out of 60 rabbits died in the first two days and were not further analyzed. Of the six dead rabbits, two were infected with $E$. coli ATCC 25922 and four were infected with $P$. aeruginosa ATCC 27853. For the survivors, no severe illness or distress occurred during the observation period. Representative examples of effect of fosfomycin on bacterial survival in the tissue-cage model are showed in Figure 2. Bacterial concentrations remained constant at ca. $1 \times 10^{9} \mathrm{CFU} \mathrm{m}^{-1}$ when rabbits were treated with saline three times daily. For treatment groups, i.v. fosfomycin was administered every $8 \mathrm{~h}$ for 3 days or 9 days, beginning at day 3 after infection. Treatment was followed by a 3 day untreated period to allow the outgrowth of residual bacteria. In both $E$. coli and $P$. aeruginosa infections, doses of fosfomycin at $30 \mathrm{mg} \mathrm{kg}^{-1}$ per day reduced bacterial numbers at first and bacteria grew quickly to the original level; at doses of fosfomycin $\geqslant 100 \mathrm{mg} \mathrm{kg}^{-1}$ per day, similar bacterial growth inhibition was detected, indicating that fosfomycin exerts non-concentration-dependent antimicrobial effects. Against $P$. aeruginosa, an initial bacterial reduction is observed at doses of fosfomycin $\geqslant 100 \mathrm{mg} \mathrm{kg}^{-1}$ per day but $\leqslant 900 \mathrm{mg} \mathrm{kg}^{-1}$ per day; however, bacterial re-growth occurred between day 2 and 3 of exposure to fosfomycin alone in the killing curves (Figure 2b). In contrast, growth inhibition of $E$. coli was observed for dose of fosfomycin $\geqslant 100 \mathrm{mg} \mathrm{kg}^{-1}$ per day, and no bacterial re-growth was observed during the late treatment and post-treatment for all doses of fosfomycin, even when the number of bacteria reduced to below detection limit on day 15 after infection (Figure 2a).

\section{Emergence of resistance in vivo}

Pharmacokinetics of fosfomycin in rabbits infected with $P$. aeruginosa, when steady-state kinetics was reached, is depicted in Figure $3 \mathrm{a}$. Samples of $P$. aeruginosa in tissue-cage fluid were examined for susceptibility to fosfomycin after treatment with various doses. Figure $3 \mathrm{~b}$ show the time courses of numbers of surviving $P$. aeruginosa cells on fosfomycin-containing agar plates at antibiotic concentrations within and out of the MSW, whereas Figure $3 \mathrm{c}$ present the concomitant changes in susceptibility. At both the lowest (group A, $30 \mathrm{mg} \mathrm{kg}^{-1}$ per day) and the highest (group F, $1200 \mathrm{mg} \mathrm{kg}^{-1}$ per day) dose of fosfomycin, no obvious change in the mutant fraction was observed (Figure 3b) and no loss in susceptibility occurred (Figure 3c). When antibiotic concentrations were inside the MSW (group B, C, D and $\mathrm{E}$ ), the population was enriched with resistant mutants on the plates with $\times 4$ the MIC of fosfomycin (Figure 3b); meanwhile, the susceptibility of bacteria decreased gradually (Figure 3c), and resistant mutants selected from group B, C, D and E showed high-fosfomycin MICs of $64-128 \mu \mathrm{g}^{-1} \mathrm{ml}$. All selection of resistant mutants occurred at day 2 after fosfomycin treatment, as indicated by both mutant fraction and MIC increases. Furthermore, the observed elevations in the mutant fraction and the MIC were stable after ten passages on antibiotic-free agar plates.

With E. coli infections, no development of resistance against fosfomycin was observed during the first 3 days treatment. Thereafter, the treatment time for E. coli infection groups was extended. Still there was no significant change both in the mutant fraction and antibiotic susceptibility in all dosage groups during the last 6 days and post-treatment period. In the meanwhile, bacteria counts 

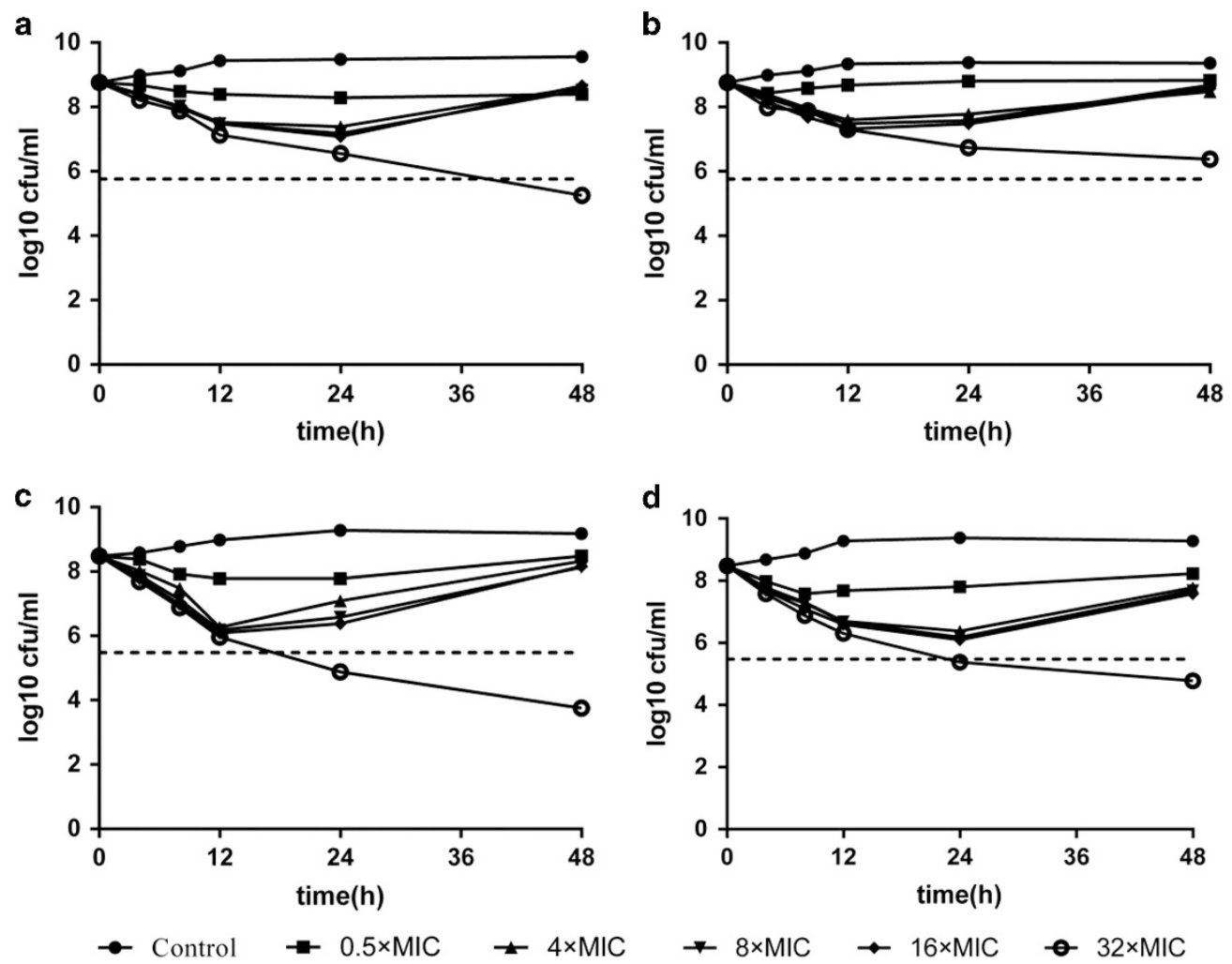

Figure 1 Time-kill curves with $0.5, \times 4, \times 8, \times 16$ and $\times 32$ the aerobic MIC of fosfomycin against Escherichia coli and Pseudomonas aeruginosa; (a) E. coli ATCC 25922 under aerobic condition, (b) P. aeruginosa ATCC 27853 under aerobic condition, (c) E. coli ATCC 25922 under anaerobic condition, (d) P. aeruginosa ATCC 27853 under anaerobic condition; Broken line, bactericidal line.
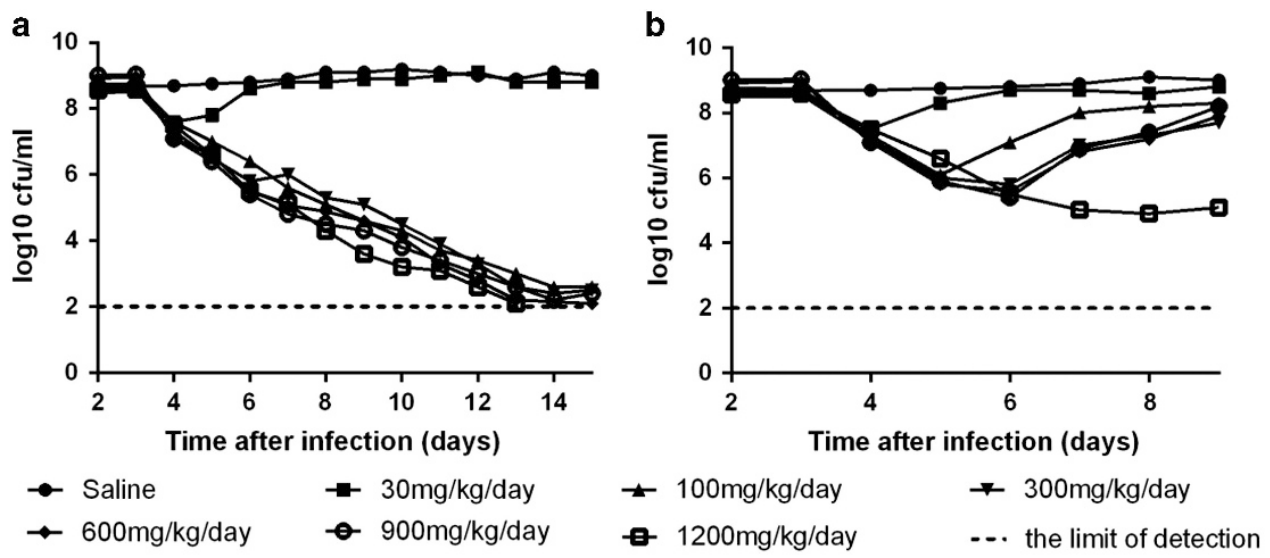

Figure 2 Effect of fosfomycin dose on bacterial survival in the tissue-cage model infected with Escherichia coli ATCC 25922 (a) and Pseudomonas aeruginosa ATCC 27853 (b). Three days after infection, various doses $\left(0,30,100,300,600,900\right.$ and $1200 \mathrm{mg} \mathrm{kg}^{-1}$ of body weight daily by use of i.v. drip) of fosfomycin were administered as 8 hourly doses for 9 days ( $E$. coll) or 3 days ( $P$. aeruginosa).

decreased to $2 \log 10 \mathrm{CFU} \mathrm{ml}^{-1}$ (the limit of detection) (Figure 2a) at day 9 after fosfomycin treatment.

Correlation of PK/PD indices with $P$. aeruginosa mutant enrichment and amplification

Comparison of MPC-based pharmacokinetic variables, determined as steady-state values after the sixth dose, with the emergence of resistant mutants are displayed in Table 2. Rabbits in group A and B whose pharmacokinetics data cannot be obtained were excluded from the
$\mathrm{PK} / \mathrm{PD}$ analysis. For no rabbit was loss of susceptibility seen when $\mathrm{AUC}_{24} / \mathrm{MPC}$ was $>10 \mathrm{~h}$, where $\mathrm{AUC}_{24}$ is the area under the drug concentration-time curve in a $24 \mathrm{~h}$ interval. When the maximum concentration (Cmax)/MPC were taken into consideration, the upper boundary of MSW was 1.8. However, when the Cmax/MPC was $>1.8$, loss of bacterial susceptibility occurred in 1 of 5 rabbits. Besides, mutants were recovered from all 15 rabbits when the fosfomycin concentration was above the MPC for $<70 \%$ of the dosing interval. Considering the fact that MPC-based PK/PD indices were reported to 

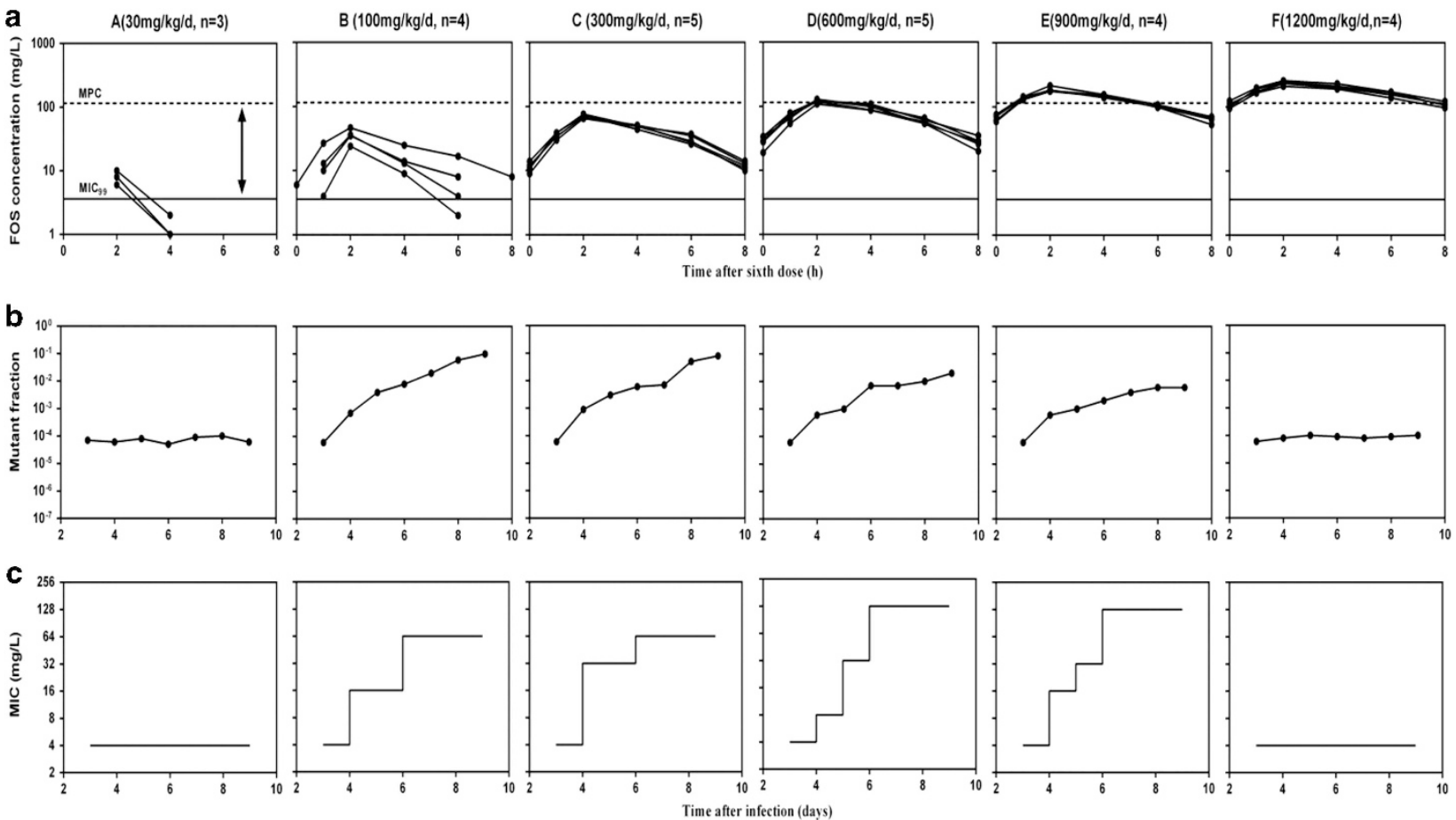

Figure 3 Effect of fosfomycin concentration in rabbit tissue-cage model of Pseudomonas aeruginosa infections on loss of susceptibility and mutant enrichment. A, B, C, D, E and F correspond to the six different treatment groups. In vivo simulated pharmacokinetics (a) was monitored at the indicated times after the administration of sixth dose. The fraction of resistant mutants (b) in each group of rabbits was determined daily as the number of colonies grown on fosfomycin-containing agar ( $\times 4$ aerobic MIC) relative to the number that grew on drug-free agar. Loss of susceptibility (c) was also monitored as an increase in MIC. The double-headed arrow indicates the mutant selection window. Some pharmacokinetics data in group A and B cannot be obtained, because concentrations were below the detection limit. d, day; FOS, fosfomycin.

Table 2 Correlation of pharmacokinetic/pharmacodynamic parameters with selection for Pseudomonas aeruginosa resistance

\begin{tabular}{|c|c|c|}
\hline PK/PD index, value ${ }^{a}$ & Fraction of rabbits with resistant bacteria & $P$-value ${ }^{b}$ \\
\hline \multicolumn{3}{|l|}{$A \cup C_{24} / M P C$} \\
\hline$>10$ & $0 / 4$ & NA \\
\hline$<10$ & $15 / 15$ & 0.001 \\
\hline \multicolumn{3}{|l|}{ Cmax/MPC } \\
\hline$>1.8$ & $2 / 5$ & 0.625 \\
\hline$<1.8$ & $12 / 14$ & 0.015 \\
\hline \multicolumn{3}{|l|}{$T>M P C$} \\
\hline$>70 \%$ & $0 / 4$ & NA \\
\hline$<70 \%$ & $15 / 15$ & 0.001 \\
\hline
\end{tabular}

\section{Abbreviations: NA, not applicable.}

High values indicate no difference from the control.

alndices were calculated using total drug concentrations.

${ }^{b} P$-values were calculated by Fisher's exact test, with a set of three infected but untreated rabbits used as a control.

be more suitable for predicting mutant-restricting antimicrobial doses than MIC-based indices, ${ }^{15,19}$ the latter indices were not shown in Table 2.

Biological fitness cost of mutants

The biological fitness of the in vitro-isolated resistant mutants was determined as the growth rate in tissue-cage fluid under aerobic and anaerobic condition. Randomly selected, single-step resistant mutants ( $n=10 ; 5 E$. coli and 5P. aeruginosa) derived from the susceptible parental strains were chosen and results for representative strains are shown in Figure 4. With E. coli, resistant mutants showed a significant slowing in their rate of growth compared with the susceptible parental strains under both aerobic and anaerobic condition. The single-step in vitro mutant strains were found to have an average doubling time of $43 \mathrm{~min}$ for susceptible strains and $84 \mathrm{~min}$ for resistant strains under aerobic condition. When grown under anaerobic conditions, the single-step mutant strains had markedly lower growth rate. The average generation time for $E$. coli was $55 \mathrm{~min}$ (susceptible strains) and $142 \mathrm{~min}$ (resistant strains). With $P$. aeruginosa, no significant difference in growth rate could be detected between the susceptible and resistant strains under either aerobic or anaerobic conditions (Figure $4 \mathrm{~b}$ ).

\section{DISCUSSION}

One particular consideration regarding the clinical application of fosfomycin is the potential for the emergence of resistance during monotherapy. ${ }^{3,6}$ As it is now widely accepted that hypoxic environments impact the activity of some classes of antibiotics including fosfomycin, we examined activity under aerobic conditions and also under anaerobic conditions which are more relevant to in vivo conditions. Our experiments showed that both E. coli ATCC 25922 and $P$. aeruginosa ATCC 27853 have high mutation frequency to fosfomycin resistance under both aerobic and anaerobic conditions (Table 1), suggesting fosfomycin-resistant mutants are easily obtained in vitro.

With the in vitro time-kill studies, fosfomycin exhibited excellent inhibiting but not killing activity against both $E$. coli and P. aeruginosa with activity greater under anaerobic conditions. These results are consistent with previous studies that have examined the activity of 

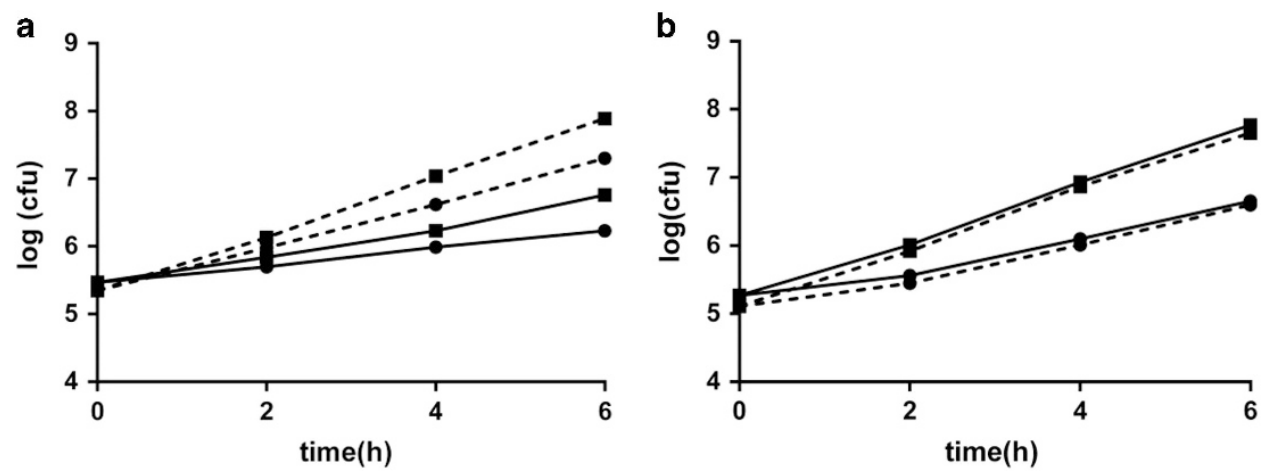

Figure 4 Growth of fosfomycin-resistant Escherichia coli (a) and Pseudomonas aeruginosa (b) (solid line) in comparison with the parental fosfomycinsusceptible strains (hidden line). The symbol used for the different experimental conditions are as follows: $\mathbf{\square}$, under aerobic condition; $\boldsymbol{\bullet}$, under anaerobic condition.

fosfomycin alone. ${ }^{20}$ Under both aerobic and anaerobic conditions, inhibitory activity of fosfomycin lasted for $12 \mathrm{~h}$ but there was re-growth thereafter for both strains at antibiotic concentrations of $\times 4, \times 8$ and $\times 16$ aerobic MIC (Figure 1 ). However, no emergence of fosfomycin resistance was detected when the antibiotic concentration below the MIC (at concentration of $\times 0.5$ aerobic MIC) or above the MPC (at concentration of $\times 32$ aerobic MIC). This phenomenon was in accord with the MSW hypothesis, which maintains that antibiotic concentrations inside the MSW are expected to allow the acquisition of resistance concurrently with eradication of susceptible pathogen populations. ${ }^{8}$ On the basis of these in vitro findings, it seems that resistance development would be extremely likely if this agent was used as monotherapy to treat infections caused by E. coli and $P$. aeruginosa, potentially leading to treatment failure. However, results from in vitro studies are often inconsistent with in vivo data.

Data from our animal model demonstrated that the selection window for fosfomycin against $P$. aeruginosa exists in vivo. Its boundaries fit well with those determined in vitro, indicating that suboptimal antibiotic dosage regimens may be a significant risk factor for emergence of resistance. It is interesting to note that the time for the mutant selected were different during therapy when drug concentration fell in a different portion of the MSW, ${ }^{9,10,12}$ but it was not observed in the present study. The mutants were selected at day 2 after fosfomycin treatment in all groups (Figure 3) and showed high-fosfomycin MIC levels $\left(32-128 \mu \mathrm{g} \mathrm{ml}^{-1}\right)$. In order to optimize dosages with respect to minimizing resistance development, we further investigated the relationship between $\mathrm{PK} / \mathrm{PD}$ parameters on the basis of the MPC concept and the emergence of resistant mutants. The results showed that when $\mathrm{AUC}_{24} / \mathrm{MPC}$ and $\mathrm{T}>\mathrm{MPC}$ were above 90 and $70 \%$, respectively, complete eradication occurred and no decrease in susceptibility was observed. However, the present work cannot identify the most predictive index, since only one organism was tested.

The rapid development of drug resistance as the major drawback of fosfomycin seems to have already become a consensus. ${ }^{3,6,21}$ With E. coli, however, the data generated in vivo did not agree with the results attained in vitro. The selection of resistant mutants of E. coli was not observed in our animal model, regardless of whether the fosfomycin concentrations were inside or outside the selection window. In our in vitro studies, E. coli ATCC 25922 compared with $P$. aeruginosa ATCC 27853 exhibited lower frequencies of mutants to fosfomycin under both aerobic and anaerobic conditions (Table 1). Overall, however, the differences seem to be marginal, and both of these two strains had a high mutation frequency to fosfomycin resistance. More importantly, the mutants of both strains with increased MIC were readily selected at the end point of the in vitro time-kill studies. So, the lower resistance mutation frequencies of E. coil are insufficient to explain the absence of resistance mutants in vivo. Actually, the apparent discrepancy between in vitro results and observations of animal studies is not haphazard. One possible mechanism is that $E$. coil often confer a biological fitness cost to the resistant bacteria that can be observed as a decreased growth rate in vitro and in vivo. ${ }^{18}$ Biological fitness is a significant determinant of bacterial survival. A mathematical model has found that such costs rapidly reduced the probability of development of fosfomycin resistance, because the resistant bacteria usually are inferior to normal bacteria and can easily be outcompeted. ${ }^{22}$ In this study, most of the in vitro-isolated resistant mutants of $E$. coli showed a decreased growth rate compared with the susceptible parental strains under both aerobic and anaerobic conditions, whereas no biological fitness cost associated with acquisition of fosfomycin resistance in $P$. aeruginosa was observed. Previous studies on the mechanism of fosfomycin resistance demonstrated that this divergence between $E$. coli and $P$. aeruginosa could be attributed to the fact that they apparently differ in the specific target genes for mutation and their metabolic implications. ${ }^{6}$

In vitro measurements cannot account for the host's immune response, which is necessary for successful recovery from infectious diseases. Recently, Handel et al. ${ }^{23}$ demonstrated that an immune response that retains its strength despite a strong drug-induced decline of bacteria numbers considerably narrows the MSW and reduces the emergence of resistance. In addition, less-fit mutants, such as fosfomycin-resistant $E$. coli mutants, are considered to be more readily eliminated by the immune system compared with the parental strain. $^{23,24}$ On the other hand, fosfomycin may have several modulatory effects on immune system function. Previous studies reported that fosfomycin enhances the phagocytic killing of invading pathogens by host cells. ${ }^{25}$ It was also able to induce the enhancement of the depressed phagocytic response of polymorphonuclear cells in immunocompromised patients. ${ }^{26}$ Above all, a brisk immune response and mechanisms responsible for biological fitness cost provide a conceivable explanation of why fosfomycin-resistant mutants could be detected in rabbits infected with $P$. aeruginosa but not with $E$. coli.

Maybe the exact mechanisms responsible for the absence of emerging resistance to fosfomycin in vivo are complicated and involve more factors. But it is interesting to note that the results of our experiment are in accordance with fosfomycin-related clinical data. In a case-control study including 113 patients with E. coli UTIs, no resistance to fosfomycin was detected and clinical cure rates were high $(93 \%) .{ }^{27}$ In contrast, the emergence of resistance to fosfomycin 
appears to be relatively frequent for systemic infections with P. aeruginosa; values between 7 and $20 \%$ have been reported in four relevant studies. ${ }^{21}$ Besides, susceptibility patterns of E. coli have remained relatively stable since the introduction of this agent in clinical practice almost 40 years ago. ${ }^{28}$ Although fosfomycin is not used as monotherapy for treatment of the $P$. aeruginosa infection, a study of 228 cases over a period of 12 years showed a significant decline in the susceptibility to fosfomycin over time. ${ }^{29}$ This probably because clinically relevant concentrations of fosfomycin combined with other antimicrobial agents enhance bacterial killing of $P$. aeruginosa, but do not suppress the emergence of fosfomycin resistance. ${ }^{30}$

Our findings may contribute little to clinical management, because clinicians use fosfomycin mainly as a combination therapy rather than as monotherapy. However, these intriguing in vitro and in vivo results suggest that the emergence of antibiotic resistance in a bacterial population is shaped by several factors. Appropriate antibiotic dosing is the key to prevent the emergence and proliferation of antibiotic-resistant strains, but it is only a part of the issue, and not even the most important. Some specific factors, such as the biological fitness costs and the host immune response, should not be ignored. Furthermore, MSW theory should not be completely disproved. However, before it can be used as a framework for the design of antimicrobial therapy, the existence of the window must be demonstrated not only in vitro but also in vivo.

\section{MATERIALS AND METHODS}

\section{Antimicrobials and chemicals}

Penicillin, as a sodium salt for injection, was purchased from Zhongnuo Pharmaceutical Group Corporation (Shijiazhuang, China). A batch of fosfomycin sodium and cis-propenylphosphonic acid (cPPA) were purchased from Northeast Pharmaceutical Factory (Shenyang, PR China). Fosfomycin standard was acquired from the National Institute for the Control of Pharmaceutical and Biological Products (Beijing, China). The N,O-bis (trimethylsilyl) trifluoracetamide with $1 \%$ trimethylchlorsilane was obtained from Sigma-Aldrich (St Louis, MO, USA). All other chemicals used in these experiments were of analytical grade.

\section{Bacterial strain and susceptibility testing}

Two standard strains of Gram-negative bacteria, those are E. coli ATCC 25922 (American Type Culture Collection, Manassas, VA) and P. aeruginosa ATCC 27853 , were selected for the study and were preserved on cryovial beads at $-80^{\circ} \mathrm{C}$ at the Anhui Center for Surveillance of Bacterial Resistance. MIC of fosfomycin was determined by agar dilution in agar medium containing $25 \mu \mathrm{g} \mathrm{ml}^{-1}$ of glucose-6-phosphate according to a standard procedure described by the Clinical and Laboratory Standards Institute in 2012. ${ }^{31}$ The measurement was followed by a second determination, plus a replicate that utilized linear drug concentration increments (10\% per sequential increase). The fraction of the colonies that were recovered was plotted against the drug concentration to determine the $\mathrm{MIC}_{99}$ by interpolation.

The MPC was determined as described elsewhere. ${ }^{32}$ Briefly, high-density cultures were prepared from overnight cultures grown in liquid medium followed by a 10 -fold dilution and $4 \mathrm{~h}$ of incubation with shaking at $35^{\circ}$ C. A series of Mueller-Hinton agar (Difco) plates containing known antibiotic concentrations and $25 \mathrm{\mu g} \mathrm{ml}^{-1}$ glucose-6-phosphate were inoculated with ca. $10^{10} \mathrm{CFU}$ of bacteria. The inoculated plates were incubated for $72 \mathrm{~h}$ at $35^{\circ} \mathrm{C}$ and screened visually for growth. Preliminary MPC was recorded as the lowest drug concentration that prevented growth. To estimate the exact MPC, logarithms of bacterial numbers were plotted against antibiotic concentrations. MPC was taken as the point where the plot intersected the theoretical limit of detection $\left(\log \mathrm{CFU} \mathrm{m}{ }^{-1}=1\right)$. MIC and MPC were determined under both aerobic and anaerobic conditions.

\section{Mutation frequency determination}

For an estimation of the in vitro spontaneous mutant frequency of E. coli and $P$. aeruginosa, 25 independent $3 \mathrm{ml}$ Luria-Bertani (LB) broth cultures were inoculated with $\sim 10^{3}$ cells and grown to saturation (ca. $3 \times 10^{9} \mathrm{CFU} \mathrm{ml}{ }^{-1}$ ) at $35^{\circ} \mathrm{C}$. Aliquots from successive dilutions or concentrated cultures were plated onto Mueller-Hinton $(\mathrm{MH})$ agar plates containing fosfomycin at $\times 4, \times 8$ or $\times 16$ aerobic MIC and $25 \mu \mathrm{g} \mathrm{ml}^{-1}$ of glucose-6-phosphate. In addition, appropriate dilutions without antibiotic were plated onto $\mathrm{MH}$ agar plates to estimate viability. The number of colonies growing after $24 \mathrm{~h}$ of aerobic or anaerobic incubation was determined. The mutation frequency was calculated from the median number of resistant cells divided by the total number of cells per milliliter of culture. MIC values were calculated for three representative spontaneous mutants and compared with those for the parental strain.

\section{In vitro time-kill studies}

In vitro bactericidal activities of fosfomycin against high-inoculum of $E$. coli and $P$. aeruginosa were assessed by modified time-kill assays. ${ }^{33}$ High-inoculum time-kill studies employed log-phase cultures which were diluted into $34 \mathrm{ml}$ (equivalent to the volume of Wiffle ball used in tissue-cage infection model) of prewarmed $35^{\circ} \mathrm{C}$ LB broth medium to yield initial cell count of $3.0 \times 10^{8}$ $\mathrm{CFU} \mathrm{ml} l^{-1}$. The drugs were added to the bacterial cultures at concentrations corresponding to $\times 0.5, \times 4, \times 8, \times 16$ and $\times 32$ aerobic MIC and $25 \mu \mathrm{g} \mathrm{ml}^{-1}$ of glucose-6-phosphate. Bacterial cultures and antibiotics were incubated at $35^{\circ} \mathrm{C}$ under aerobic or anaerobic conditions. Drug-free flasks were included as controls. Surviving bacteria were counted after $0,4,8,12,24$ and $48 \mathrm{~h}$ of incubation at $35^{\circ} \mathrm{C}$ by subculturing $100 \mu \mathrm{l}$ serial dilution of every flask on $\mathrm{MH}$ agar plates. The lower limit of accurate and reproducible detectable colony count was $2 \log _{10} \mathrm{CFU} \mathrm{ml} l^{-1}$. Each in vitro experiments was performed at least three times.

\section{Tissue-cage infection model}

Healthy female New Zealand rabbits (weight, $2.5-3 \mathrm{~kg}$ ) were provided by the Anhui Medical University Experimental Animal Center. The rabbits were housed individually according to the Guide for the Care and Use of Laboratory Animals. ${ }^{34}$ After the rabbits were anesthetized by use of sodium pentobarbital $\left(30 \mathrm{mg} \mathrm{kg}^{-1}\right.$ ) intramuscularly, a plastic ball (Wiffle ball, $43 \mathrm{~mm}$ in diameter, with a volume of $34 \mathrm{ml}$ ) was implanted subcutaneously into each rabbit through a dorsal midline incision under aseptic conditions. For preventing secondary infection caused by the implantation surgery, penicillin (100 $000 \mathrm{IU} \mathrm{kg}^{-1}$ ) was administrated intramuscularly to rabbits twice a day for 3 days. By 4-6 weeks after implantation, each plastic ball were sealed by connecting tissues and filled with clear tissue fluid. ${ }^{35}$ Approximately $3.0 \times 10^{10}$ CFU E. coli or P. aeruginosa culture was concentrated in $1 \mathrm{ml}$ of $0.9 \% \mathrm{NaCl}$ and injected into each pre-implanted plastic ball. Two days after infection, $0.5 \mathrm{ml}$ of tissue-cage fluid was withdrawn from each plastic ball for a viable bacteria count. Rabbits having $>3 \times 10^{8} \mathrm{CFU} \mathrm{ml}^{-1}$ viable bacterial cells were treated with various doses of fosfomycin. The experimental protocol was approved by the Committee on the Ethics of Animal Experiments of Anhui Medical University (permit number 13-0406).

\section{Simulated pharmacokinetic profiles}

According to the results of the prior experiment, rabbits infected with $P$. aeruginosa were treated with fosfomycin (as three 8 hourly doses) for 3 consecutive days, whereas E. coli infection groups received longer-duration treatment (9 days). Rabbits were administered fosfomycin at $0 \quad(n=6)$, $30(n=6), 100(n=8), 300(\mathrm{n}=9), 600(n=9), 900(n=8)$ and $1200(n=8)$ $\mathrm{mg} \mathrm{kg}^{-1}$ of body weight daily by use of an i.v. drip beginning at 3 days after infection. These rabbits were randomly assigned to each treatment group in order to provide fosfomycin pharmacokinetics at concentrations below the $\mathrm{MIC}_{99}$, between the $\mathrm{MIC}_{99}$ and MPC, and above the MPC.

\section{Pharmacokinetic measurements}

Tissue-cage fluid $(0.5 \mathrm{ml})$ was collected from each plastic ball at $0,1,2,4,6$ and $8 \mathrm{~h}$ after each fosfomycin dose. Fluid samples were clarified by centrifugation at $8900 \mathrm{~g}$ for $10 \mathrm{~min}$ and stored at $-80^{\circ} \mathrm{C}$. The total drug concentrations of tissue-cage fluid samples were determined by a previously published but 
modified GC method. ${ }^{36}$ Samples were precipitated with four volumes of methanol containing $200 \mu \mathrm{g}$ of cPPA per $\mathrm{mL}$ as the internal standard and were centrifuged at $16000 \mathrm{~g}$ for $5 \mathrm{~min}$ at room temperature. A $100 \mu \mathrm{l}$ sample of the supernatant was evaporated to dryness under a gentle stream of nitrogen and was derivatized with $\mathrm{N}, \mathrm{O}-\mathrm{bis}$ (trimethylsilyl) trifluoracetamide with $1 \%$ trimethylchlorsilane for $15 \mathrm{~min}$ at $56^{\circ} \mathrm{C}$. A $1 \mu \mathrm{l}$ aliquot of the silylated solution was applied to the gas chromatograph.

GC analysis was performed using an Agilent 7890A (Agilent Technologies, Shanghai, China), which was coupled to a flame ionization detector equipped with a capillary and HP-5 column $(30 \mathrm{~m}$ long $\times 0.32 \mathrm{~mm}$ i.d.) coated with a $0.25 \mathrm{pm}$ film of phenylmethylsiloxane. The injector and detector temperatures were set at 250 and $270{ }^{\circ} \mathrm{C}$, respectively. The oven temperature program used in the analysis had an initial temperature of $150^{\circ} \mathrm{C}$ that was held for $3 \mathrm{~min}$, and then ramped at $10^{\circ} \mathrm{C} \mathrm{min}-1$ to $250^{\circ} \mathrm{C}$, and held for $2 \mathrm{~min}$.

Data acquisition was performed with the HP Chem program (Agilent). The relation between the ratio of the peak area of fosfomycin and the peak area of the internal standard and the concentrations of fosfomycin were linear, with a correlation coefficient of 0.9975 . The coefficients of variation were $7.7 \%$ at $10 \mu \mathrm{g} \mathrm{ml}^{-1}, 6.3 \%$ at $50 \mu \mathrm{g} \mathrm{ml}^{-1}$ and $3.1 \%$ at $200 \mu \mathrm{g} \mathrm{ml}^{-1}(n=6)$. Intra-day and inter-day coefficients of variations were $<5 \%$. The limit of detection was $1 \mu \mathrm{g} \mathrm{ml}^{-1}$.

\section{Counts of bacteria}

In each animal experiment, surviving bacteria were counted every $24 \mathrm{~h}$ of incubation at $35^{\circ} \mathrm{C}$ by subculturing $100 \mu \mathrm{l}$ serial dilution of every plastic ball on $\mathrm{MH}$ agar plates. In order to account for antibiotic carryover, all samples were diluted sufficiently before plating, therefore reducing the antibiotic concentration below the MIC of the drug. The lower limit of accurate detection was $2 \log _{10} \mathrm{CFU} \mathrm{ml}{ }^{-1}$.

\section{Detection of fosfomycin-resistant mutants}

Loss of susceptibility was monitored in LB broth at the end point of the in vitro time-kill studies and in tissue-cage fluid obtained daily before fosfomycin administration, during antibacterial treatment, and 24 and $48 \mathrm{~h}$ after the termination of fosfomycin treatment. After counting CFU on drug-free plates to determine treatment efficacy, an attempt was made to scrape together all colonies from the most heavily populated plates. This material was resuspended in $7 \mathrm{ml}$ normal saline to obtain an approximate cell density of $1 \times 10^{8} \mathrm{CFU} \mathrm{ml}^{-1}$ and replated on $\mathrm{MH}$ agar plates (supplemented with $25 \mu \mathrm{g} \mathrm{ml}^{-1}$ of glucose-6phosphate) with and without $\times 4$ MIC of fosfomycin. Samples were considered to be enriched for drug-resistant mutants if the proportion of resistant CFU observed was $\geqslant 1 \log _{10}$ greater than the baseline proportion observed in untreated rabbits at day $0 .{ }^{9}$ The MICs of fosfomycin for bacteria isolated from plastic ball were determined before and after antimicrobial exposure by using the standard method described above. Moreover, stability of the observed resistance was determined daily by consecutive passaging of bacteria on antibiotic-free agar plates for 10 consecutive days.

\section{Biological fitness cost of mutants}

The biological fitness cost associated with the development of fosfomycin resistance was determined by comparing the growth of the resistant strain with that of the susceptible parent strain using growth curves. ${ }^{20}$ Selection of resistant mutants were obtained by plating the high inocula (ca. $10^{10} \mathrm{CFU}$ ) of parental strains on $\mathrm{MH}$ agar plates containing $25 \mu \mathrm{g} \mathrm{ml}^{-1}$ of glucose-6-phosphate and $\times 8$ MIC of fosfomycin, and MICs were determined to confirm resistance. Flasks containing $50 \mathrm{ml}$ of fresh LB broth were inoculated with $0.5 \mathrm{ml}$ of an overnight broth culture of the pathogen to be tested. Subcultures were grown in tissue-cage fluid under aerobic or anaerobic conditions. Appropriate dilutions of cultures were plated on $\mathrm{MH}$ agar medium every $2 \mathrm{~h}$. After overnight incubation at $35{ }^{\circ} \mathrm{C}$ colonies were counted and the generation time was calculated.

\section{Statistical analysis}

Fisher's exact test was used for statistical analysis of the pharmacokinetic/ pharmacodynamic data, with an infected but untreated set of rabbits as a control. $P<0.01$ was considered to be statistically significant.

\section{CONFLICT OF INTEREST}

The authors declare no conflict of interest.

\section{ACKNOWLEDGEMENTS}

This work was supported by the National Natural Science Foundation of China (no 81071394).

1 Pitout, J. D., Chan, W. W. \& Church, D. L. Tackling antimicrobial resistance in lower urinary tract infections: treatment options. Expert Rev. Anti. Infect. Ther. 24, 1-12 (2016).

2 Monurol package insert. St Louis, MO, Forest Pharmaceuticals, Inc.; (1997). http:// druginserts.com/lib/rx/meds/monurol-1/.

3 Falagas, M. E., Giannopoulou, K. P., Kokolakis, G. N. \& Rafailidis, P. I. Fosfomycin: use beyond urinary tract and gastrointestinal infections. J. Antimicrob. Chemother. 46, 1069-1077 (2008)

4 Walsh, C. C, McIntosh, M. P., Peleg, A. Y., Kirkpatrick, C. M. \& Bergen, P. J. In vitro pharmacodynamics of fosfomycin against clinical isolates of Pseudomonas aeruginosa. J. Antimicrob. Chemother. 70, 3042-3050 (2015).

5 Gómez-Garcés, J. L., Gil-Romero, Y., Sanz-Rodríguez, N., Muñoz-Paraíso, C. \& Regodón-Domínguez, M. In vitro activity of fosfomycin, alone or in combination, against clinical isolates of carbapenem resistant Pseudomonas aeruginosa. Enferm. Infecc. Microbiol. Clin. 34, 228-231 (2016).

6 Rodríguez-Rojas, A. et al. Assessing the emergence of resistance: the absence of biological cost in vivo may compromise fosfomycin treatments for $P$. aeruginosa infections. PLOS ONE 5, e10193 (2010).

7 Epstein, B. J, Gums, J. G. \& Drlica, K. The changing face of antibiotic prescribing: the mutant selection window. Ann. Pharmacother. 38, 1675-1682 (2004).

8 Zhao, X. \& Drlica, K. Restricting the selection of antibiotic-resistant mutants: a general strategy derived from fluoroquinolone studies. Clin. Infect. Dis. 33(Suppl 3); S147-S156 (2001).

9 Cui, J et al. The mutant selection window in rabbits infected with Staphylococcus aureus. J. Infect. Dis. 194, 1601-1608 (2006).

$10 \mathrm{Ni}$, W., Song, X. \& Cui, J. Testing the mutant selection window hypothesis with Escherichia coli exposed to levofloxacin in a rabbit tissue cage infection model. Eur. J. Clin. Microbiol. Infect. Dis. 33, 385-389 (2014).

$11 \mathrm{Li}, \mathrm{X}$. et al. Evaluation of meropenem regimens suppressing emergence of resistance in Acinetobacter baumannii with human simulated exposure in an in vitro intravenous-infusion hollow-fiber infection model. Antimicrob. Agents Chemother. 58, 6773-6781 (2014)

12 Zhu, Y. L. et al. Testing the mutant selection window in rabbits infected with methicillin-resistant Staphylococcus aureus exposed to vancomycin. J. Antimicrob. Chemother. 67, 2700-2706 (2012).

13 Hesje, C. K., Drlica, K. \& Blondeau, J. M. Mutant prevention concentration of tigecycline for clinical isolates of Streptococcus pneumoniae and Staphylococcus aureus. J. Antimicrob. Chemother. 70, 494-497 (2015).

14 Cui, J. C., Liu, Y. N. \& Chen, L. A. Mutant prevention concentration of tigecycline for carbapenem-susceptible and -resistant Acinetobacter baumannii. J. Antibiot. 63, 29-31 (2010).

15 Liang, B. et al. Mutant prevention concentration-based pharmacokinetic/pharmacodynamic indices as dosing targets for suppressing the enrichment of levofloxacin-resistant subpopulations of Staphylococcus aureus. Antimicrob. Agents Chemother. 55, 2409-2412 (2011).

16 Liu, L. G. et al. Comparative study of the mutant prevention concentrations of vancomycin alone and in combination with levofloxacin, rifampicin and fosfomycin against methicillin-resistant Staphylococcus epidermidis. J. Antibiot. 66, 709-712 (2013).

$17 \mathrm{Mei}, \mathrm{Q}$ et al. Use of Monte Carlo simulation to evaluate the development of vancomycin resistance in meticillin-resistant Staphylococcus aureus. Int. J. Antimicrob. Agents 45, 652-656 (2015)

18 Olofsson, S. K. \& Cars, O. Optimizing drug exposure to minimize selection of antibiotic resistance. Clin. Infect. Dis. 45(Suppl 2); S129-S136 (2007).

19 Drlica, K., Zhao, X., Blondeau, J. M. \& Hesje, C. Low correlation between MIC and mutant prevention concentration. Antimicrob. Agents Chemother. 50, 403-404 (2006).

20 McCaughey, G., Diamond, P., Elborn, J. S., McKevitt, M. \& Tunney, M. M. Resistance development of cystic fibrosis respiratory pathogens when exposed to fosfomycin and tobramycin alone and in combination under aerobic and anaerobic conditions. PLOS ONE 8, e69763 (2013).

21 Karageorgopoulos, D. E., Wang, R., Yu, X. H., McKevitt, M. \& Tunney, M. M. Fosfomycin: evaluation of the published evidence on the emergence of antimicrobial resistance in Gram-negative pathogens. J. Antimicrob. Chemother. 67, 255-268 (2012).

22 Nilsson, A. I., Berg, O. G., Aspevall, O., McKevitt, M. \& Tunney, M. M. Biological costs and mechanisms of fosfomycin resistance in Escherichia coli. Antimicrob. Agents Chemother. 47, 2850-2858 (2003).

23 Handel, A., Margolis, E. \& Levin, B. R. Exploring the role of the immune response in preventing antibiotic resistance. J. Theor. Biol. 256, 655-662 (2009) 
24 Schulz zur Wiesch, P., Engelstädter, J. \& Bonhoeffer, S. Compensation of fitness costs and reversibility of antibiotic resistance mutations. Antimicrob. Agents Chemother. $\mathbf{5 4}$ 2085-2095 (2010).

25 Pérez Fernández, P., Herrera, I., Martínez, P., Gómez-Lus, M. L. \& Prieto, J. Enhancement of the susceptibility of Staphylococcus aureus to phagocytosis after treatment with fosfomycin compared with other antimicrobial agents. Chemotherapy 41, 45-49 (1995).

26 Tullio, V. et al. Role of fosfomycin tromethamine in modulating non-specific defence mechanisms in chronic uremic patients towards ESBL-producing Escherichia coli. Int. J. Immunopathol. Pharmacol. 21, 153-160 (2008).

27 Rodriguez-Bano, J. et al. Community infections caused by extended-spectrum beta-lactamase-producing Escherichia coli. Arch. Intern. Med. 168, 1897 (2008).

28 Vardakas, K. Z., Legakis, N. J., Triarides, N. \& Falagas, M. E. Susceptibility of contemporary isolates to fosfomycin: a systematic review of the literature. Int. J. Antimicrob. Agents 47, 269-285 (2016).

29 Rodríguez López, F. C., Franco-Alvarez de Luna, F., Gordillo Urbano, R. M., Ibarra González, A. \& Casal Román, M. Microorganisms isolated from outpatient urine samples and antimicrobial susceptibility over a 12-year period. Rev. Esp. Quimioter. 18, 159-167 (2005).

30 Walsh, C. C. et al. Clinically relevant concentrations of fosfomycin combined with polymyxin $\mathrm{B}$, tobramycin or ciprofloxacin enhance bacterial killing of Pseudomonas aeruginosa, but do not suppress the emergence of fosfomycin resistance. J. Antimicrob. Chemother. 71, 2218-2229 (2016).

31 Clinical and Laboratory Standards Institute. Performance standards for antimicrobial susceptibility testing; twenty-second informational supplement. CLSI document M100-S22 32, 1-188 (2012).

32 Zhao, X. \& Drlica, K. Restricting the selection of antibiotic-resistant mutant bacteria: measurement and potential uses of the mutant selection window. J. Infect. Dis. 185, 561-565 (2002).
33 Robertson, G. T. et al. In vitro evaluation of CBR-2092, a novel rifamycinquinolone hybrid antibiotic: microbiology profiling studies with staphylococci and streptococci. Antimicrob. Agents Chemother. 52, 2324-2334 (2008).

34 National Research Council. Guide for the care and use of laboratory animals 7th ed. Washington (DC): National Academies Press (1996).

35 Fernandez, J., Barrett, J. F., Licata, L., Amaratunga, D. \& Frosco, M. Comparison of efficacies of oral levofloxacin and oral ciprofloxacin in a rabbit model of a staphylococcal abscess. Antimicrob. Agents Chemother. 43, 667-671 (1999).

36 Dios-Vieitez, M. C., Goni, M. M., Renedo, M. J. \& Fos, D. Determination of fosfomycin in human urine by capillary gas chromatography: application to clinical pharmacokinetic studies. Chromatographia 43, 293-295 (1996).

(c) (i) () $\ominus$ This work is licensed under a Creative Commons Attribution-NonCommercial-NoDerivs $\quad 4.0$ Inter-

national License. The images or other third party material in this article are included in the article's Creative Commons license, unless indicated otherwise in the credit line; if the material is not included under the Creative Commons license, users will need to obtain permission from the license holder to reproduce the material. To view a copy of this license, visit http://creativecommons.org/licenses/bync-nd/4.0/

(C) The Author(s) 2017 